\title{
Treatment of Hydatid Liver Disease: What does the evidence say
}

\author{
Tariq A Gojwari, M.D., Tahleel A Shera, M.D., Naseer A Choh, M.D., Feroze A Shaheen, M.D., \\ Manjeet Singh, M.D., Irfan Robbani, M.D., Sheikh Reyaz, M.D.
}

Department of Radiodiagnosis and Imaging, SKIMS, Soura, Srinagar

Cystic echinococcosis is a disease of animals and humans caused by the larval stage of of Echinococcus granulosus sensu lato and has a substantial public health impact globally. The condition is considered a neglected tropical disease (NTD) and neglected zoonotic disease (NZD), and prioritized by the World Health Organization (WHO) ${ }^{1}$. Within the last 10 years, advancements have taken place in Echinococcus biology and genetics, including the delineation of new species within E. granulosus sensu lato $(\text { s.l. })^{2,3}$ and full sequencing of E. granulosus sensustricto ${ }^{2}$. Consequently, researchers are now provided with new tools to better understand parasite biology and host-parasite interactions, with the goal of opening new avenues for therapy ${ }^{2}$. Echinococcus granulosus sensu stricto (s. s.) (G13 ) is responsible for the great majority of human $\mathrm{CE}$ worldwide $(88.48 \%)$, has the most cosmopolitan distribution and is often associated with transmission via sheep as intermediate hosts ${ }^{4}$

Clinical manifestations of human CE typically result from the growth of single or multiple cysts in the liver, lungs, and/or other organs that can eventually produce a mass effect and impair organ function. The diagnosis and clinical management of cystic echinococcosis have evolved over decades in the absence of a systematic approach ${ }^{11,12}$. The first widely used ultrasound classification of hepatic cystic echinococcosis cysts has been proposed by Gharbi et al. in $1981^{5}$ and has been used in the following years as the basis for a number of other classifications. The heterogeneity of these classifications resulted in the impossibility to compare the observations and treatment protocols made by

\section{Correspondence}

Dr. Tahleel Altaf Shera, M.D.

Senior Resident

Department of Radiodiagnosis SKIMS

E-mail : tahleel149@yahoo.co.in investigators in various clinical and epidemiological settings, which were not reported in a uniform manner. To overcome this problem, in 2003, the WHO-IWGE issued a standardized classification of hepatic cystic echinococcosis cysts 'to facilitate both the uniform reporting of results from field epidemiological studies as well as in clinical studies conducted in different parts of the world, which could be easy to use in different settings and could reflect physiopathological mechanisms of cystic echinococcosis ${ }^{6}$. WHO-IWGE classification introduced the grouping of cystic echinococcosis cysts into three clinical categories: active, transitional, and inactive, reflecting the current knowledge of the natural history of cystic echinococcosis (CE). Accordingly, unilocular cysts (CE1) and multiloculated cysts with daughter vesicles without solid matrix (CE2) were classified as active cysts; cysts with detached endocyst and multiloculated cysts with daughter vesicles in a solid matrix were classified in the transitional group (CE3); and cysts with solid content with (CE5) or without (CE4) calcifications were classified as inactive cysts ${ }^{6}$.

Treatment usually consists of either a watch-and-wait approach, albendazole administration, surgical, percutaneous or per-endoscopic intervention, depending on the stage, integrity, number, size, and location of cysts ${ }^{7}$. The clinical management of cystic echinococcosis performed by different centres is extremely heterogeneous. In the past, surgery has been the only treatment modality for cystic echinococcosis, but with the introduction of benzimidazoles from the mid-1970s $\mathrm{s}^{8,9}$ and the development of percutaneous treatment in the mid-1980s ${ }^{10}$, new options became available and were widely used in the following decades. Furthermore, the so-called 'watch and-wait' approach to uncomplicated inactive cysts has also been introduced on the basis of the observation that a good 
proportion of cysts become spontaneously inactive without any treatment and such cysts are likely to remain stable over time $^{11}$. No 'one-size-fits-all' treatment for cystic echinococcosis exists because of the proteiform presentation and evolution of this infection. Moreover, in the vast majority of cases, active hepatic infection is an asymptomatic disease with a spontaneous benign evolution . However, the accumulated knowledge based on singlecentre experiences and on the few clinical trials comparing at least some of these treatment modalities ${ }^{12,16}$ supports a stage-specific approach to hepatic cystic echinococcosis ${ }^{7,11}$. The WHO-IWGE expert consensus for the diagnosis and treatment of echinococcosis in humans, issued in 2010, indicated several stage-specific options for the management of hepatic cystic echinococcosis, although it also acknowledged that the choice of treatment for individual patients depends on a number of variables, including not only cyst stage but also other characteristics of the cyst, the patient, and the centre where the patient is visited. Additional important advancements introduced with this document concern the agreement on the use of benzimidazoles. These should be used as a peri-operative adjuvant to prevent iatrogenic seeding leading to local or systemic/extrahepatic secondary echinococcosis. Furthermore, when used as the only treatment, they should be administered continuously, without the 2-week interruption between monthly cycles recommended previously. Moreover, the need for a long-term follow-up of cystic echinococcosis patients was also stated, although a definite length in years could not be provided

Over the last decade, a structured cyst stage-specific approach to patient management has been recommended based on the WHO-International Working Group on Echinococcosis cyst classification scheme, which was officially adopted in $2010^{7}$. Globally, there has been a push for physicians to take a patient's clinical condition, technical capability of the healthcare facility, safety and effectiveness of the approach, and the cost of treatment into account when devising a treatment plan. Unfortunately prospective studies to guide evidence-based therapeutic strategies are lacking and the adoption of a stage-specific approach by physicians has been slow ${ }^{17}$.In addition, whatever treatment plan is selected, long-term follow-up should be mandatory in order to detect recurrences ${ }^{7,17}$.

\section{General indications for treatment: a stage-specific approach}

The opinion of WHO-IWGE experts ${ }^{7}$ with regard to a stagespecific approach is summarized in Table 1. Radical surgery aims to remove cysts completely. Percutaneous treatments (PT) and antiparasitic treatment with benzimidazoles (BMZ) represent alternatives to surgery. Cyst type (according to US classification), size, location and presence/absence of complications are the basis for decision-making ${ }^{18}$. The present quality of evidence and strength of recommendation has been characterized as per . Infectious Diseases Society of America grading system in Table 2

Table 1. Suggested stage-specific approach to uncomplicated cystic echinococcosis of the liver ${ }^{\mathrm{a}}$.

\begin{tabular}{|c|c|c|c|c|c|}
\hline WHO classification & Surgery & Percutaneous treatment & Drug therapy & Suggested & Resources setting \\
\hline \multirow[t]{4}{*}{ CE1 } & & & & $<5 \mathrm{~cm} \mathrm{ABZ}$ & Optimal \\
\hline & & & & PAIR & Minimal \\
\hline & & $\checkmark$ & $\checkmark$ & $>5 \mathrm{~cm}$ PAIR $+\mathrm{ABZ}$ & Optimal \\
\hline & & & & PAIR & Minimal \\
\hline \multirow[t]{3}{*}{ CE2 } & & & $\checkmark$ & Other PT + ABZ & Optimal \\
\hline & $\checkmark$ & $\checkmark$ & & & \\
\hline & & & & Other PT & Minimal \\
\hline \multirow[t]{4}{*}{ CE3a } & & & & $<5 \mathrm{~cm} \mathrm{ABZ}$ & Optimal \\
\hline & & $\checkmark$ & $\checkmark$ & PAIR & Minimal \\
\hline & & & & $>5 \mathrm{~cm}$ PAIR $+\mathrm{ABZ}$ & Optimal \\
\hline & & & & PAIR & Minimal \\
\hline \multirow[t]{3}{*}{ CE3b } & & & & Non-PAIR PT + ABZ & Optimal \\
\hline & $\checkmark$ & $\checkmark$ & $\checkmark$ & & \\
\hline & & & & Non-PAIR PT & Minimal \\
\hline CE4 & & & & Watch and Wait & Optimal \\
\hline CE5 & & & & Watch and Wait & Optimal \\
\hline
\end{tabular}


Table 2. Infectious Diseases Society of America grading system (strength of recommendation and quality of evidence).

\begin{tabular}{|l|l|}
\hline \multicolumn{2}{|l|}{ Strength of recommendation } \\
\hline A & Good evidence to support a recommendation for use \\
\hline B & Moderate evidence to support a recommendation for use \\
\hline C & Poor evidence to support a recommendation \\
\hline D & Moderate evidence to support a recommendation against use \\
\hline E & Good evidence to support recommendation against use \\
\hline \hline Quality of evidence \\
\hline I & Evidence from $\geq 1$ properly randomized, controlled trial \\
\hline II & $\begin{array}{l}\text { Evidence from } \geq 1 \text { well-designed clinical trial, without randomization; from cohort or case-controlled analytic } \\
\text { studies; from multiple time series; or from dramatic results from uncontrolled experiments }\end{array}$ \\
\hline III & $\begin{array}{l}\text { Evidence from opinions of respected authorities, based on clinical experience, descriptive studies, or reports of } \\
\text { committees }\end{array}$ \\
\hline
\end{tabular}

\section{SURGERY}

The most common technique is total or partial cystectomy. Although surgical techniques have improved, there is considerable controversy as to what is the most effective technique, the role of cyst aspiration and external drainage, hepatic and lung resection, management of the residual cavity in CE of the liver, cyst recurrence after surgery, and high rates of complications and mortality related to reoperation in recurrent disease (1924). An immediate cure is claimed for surgical treatment of liver cysts, but even with radical procedures, this is far from being achieved, with morbidity, mortality, and relapse rates of $32 \%, 8 \%$, and $20 \%$, respectively. Surgery should be carefully evaluated against other options before choosing this treatment. It is the first choice for complicated cysts. In the liver, it is indicated for:1 removal of large CE2-CE3b cysts with multiple daughter vesicles, 2 single liver cysts, situated superficially, that may rupture spontaneously or as a result of trauma when PTs are not available, 3 infected cysts, again, when PTs are not available, 4 cysts communicating with the biliary tree (as alternative to PT) and5 cysts exerting pressure on adjacent vital organs. Surgery can be performed as an open procedure, with either radical or conservative techniques, or laparoscopically. There are still controversies as to the safest and most effective technique, and in which cases it should be applied ${ }^{25,27}$. As a rule, perioperative $\mathrm{ABZ}$ prophylaxis, from $1 \mathrm{wk}$ prior to surgery until $4 \mathrm{wk}$ postoperatively, is necessary to minimize the risk of secondary echinococcosis from seeding of protoscoleces in the abdominal cavity ${ }^{7}$.
Recurrence, both local and as secondary echinococcosis, is associated with spillage during removal of the cyst, incomplete removal of the endocyst, and possibly the presence of unnoticed exophytic cyst development ${ }^{28,29}$. For the latter, intraoperative US has been shown to be an important tool to improve the quality of hepatic surgery ${ }^{30}$.

\section{Contraindications}

Surgery is contraindicated in patients to whom general contraindications for surgery apply, inactive asymptomatic cysts, difficult to access cysts, and very small cysts

Strength of recommendation: B Quality of Evidence: III

\section{Percutaneous treatment}

This includes PAIR and its modifications. PAIR is a minimally invasive technique used in the treatment of cysts in the liver and other abdominal locations. Best results with PAIR + BMZ are achieved in $>5 \mathrm{~cm} \quad \mathrm{CE} 1$ and $\mathrm{CE} 3$ acystswhere it may be the first-line treatment. It is also indicated for inoperable patients and those who refuse surgery, in cases of relapse after surgery or failure to respond to BMZ alone ${ }^{12,14,16}$. Communication with bile ducts should be assessed by analyzing cyst fluid for bilirubin or by injecting contrast medium into the cyst cavity before the "injection" step ${ }^{31}$. Giant $(>10 \mathrm{~cm})$ cysts are best treated with continuous catheter drainage until the daily output falls below $10 \mathrm{~mL}^{32}$. In pregnant women with symptomatic cysts and in children aged $<3$ years, the risk of BMZ must be carefully assessed ${ }^{33}$.Protoscolicides used in PAIR are mainly $20 \% \mathrm{NaCl}$ and $95 \%$ ethanol. Transhepatic cyst puncture prevents peritoneal protoscolex spillage. 
Prophylaxis with ABZ $4 \mathrm{~h}$ before and 1 month after PAIR is mandatory ${ }^{34}$. Percutaneous treatment procedures may be performed on an out-patient basis ${ }^{35}$; our department routinely performs this procedure under day-care admission with most patients being discharged after a few hours of observation.

Puncture of echinococcal cysts had long been discouraged because of the risk of anaphylactic shock and spillage of the fluid; however, as experience with US-guided interventional techniques has increased since the early 1980 s, a growing number of articles have reported its safety in treating abdominal, especially liver, echinococcal cysts. In a recent systematic analysis on percutaneous aspiration of echinococcal cysts with 5943 punctures in 5517 hepatic and non-hepatic echinococcal cysts., only 2 cases of lethal anaphylaxis $(0.04 \%)$ and 99 reversible anaphylactic reactions $(1.8 \%)$ were reported, and incidence no greater than sporadic drug related anaphylaxis ${ }^{36}$.

\section{Contraindications}

PAIR is contraindicated for CE2 and CE3b, for CE4 and CE5, and for lung cysts. Biliary fistulae contraindicate protoscolicide use.

\section{Strength of recommendation: B Quality of Evidence: III}

\section{Antiparasitic drug treatment}

While both albendazole and MBZ have been proven effective against the larval stage of E. granulosus, ABZ is the current treatment of choice due to better absorption ${ }^{37}$. $\mathrm{ABZ}$ is administered orally at a dose of $10-15 \mathrm{mg} / \mathrm{kg}$ per day continuously without interruptions, generally for 3-6 mo. However, the optimal dose and duration of treatment with ABZ have not been formally assessed.In miltiple series, reported outcome rates for hepatic cysts are: $28.5 \%-58 \%$ cure/marked improvement, $10 \%-51 \%$ partial response, $13 \%-37 \%$ no change, and $4 \%-33 \%$ worsened. Relapse rates range from $9 \%-25 \%$, and, although responsive to subsequent treatments, cysts tend to relapse multiple times $^{38}$. BMZ are indicated for inoperable patients with liver or lung CE; patients with multiple cysts in two or more organs, or peritoneal cysts. Small $(<5 \mathrm{~cm}) \mathrm{CE} 1$ and CE3a cysts in the liver and lung respond favourably to BMZ alone $^{39,40}$. BMZ should be used to prevent recurrence following surgery or PAIR ${ }^{41}$. A recent study has shown enhanced efficacy of ABZ when co-administered with Metformin when used in experimental mice for both primary and prevention of secondary hydatidosis ${ }^{42}$.

\section{Contraindications}

BMZ are contraindicated in cases with hypersensitivity to the drug, cysts at risk of rupture and in early pregnancy. $\mathrm{ABZ}$ has been proven teratogenic in rats and rabbits. Physiological exposure to ABZ and its principal metabolite, ABZ sulfoxide, in early human pregnancy is substantially lower (perhaps 10/100 times) than in the animal species in which teratogenic or embryotoxic effects have been recorded. Despite the fact that no abnormal birth outcome has been observed following ABZ administration during pregnancy, treatment of gravid or potentially gravid females should be avoided, unless the benefit of treatment significantly outweighs the potential risk to the developing fetus ${ }^{43}$.

BMZ must be used with caution in patients with chronic hepatic disease and avoided in those with bone-marrow depression. Inactive or calcified asymptomatic cysts should not be treated unless they are complicated.

$\mathrm{BMZ}$ alone are not effective in large cysts (over $10 \mathrm{~cm}$ ), as their effect is extremely slow in cysts with large volumes of fluid.

\section{Strength of recommendation: B Quality of Evidence: III}

\section{Watch and Wait approach}

Some cysts do not require any treatment if uncomplicated, namely, CE4 and CE5 (CL cysts should not be treated, until their parasitic nature has been proven). Long-term followup of patients with US imaging has increased clinicians' confidence that in selected cases, i.e. when inactive cysts are not complicated, treatment can be put on hold ${ }^{11}$.Analysis of long-term follow-up data from a prospective CE patient cohort of 223 patients which included included 30 patients with 46 inactive cysts who never received medical treatment and were managed with the wait and watch approach. No relapse was seen in this subset of patients during 18 months of follow-up. In contrast cysts which reach inactivity through treatment with benzimidazoles behaved differently,showing a relapse rate of 50\% shown in the Kaplan-Meier analysis ${ }^{44}$.

Strength of recommendation: B Quality of Evidence: III Clinical Decision-making on Hepatic CE

$\mathrm{CE}$ can be very difficult to treat and even more difficult to cure for a number of reasons. The disease is complex and dynamic, with an evolving phase and quietly growing cysts, 
followed by an involution process during which the parasite is gradually dying, leaving behind a solidified, often calcified cyst or a scar.

Each successive active cyst stage carries its own risks for serious and even life-threatening complications. This variation during the $\mathrm{CE}$ disease process leads to a wide range of treatment modalities with an equally wide range of technological and training backgrounds necessary for implementation and delivery. As a result of all of these issues, no "one size fits all" management approach is available, and a stage-specific approach currently appears to be the best way to manage this condition.

Control program implementation for CE has historically relied on a combination of local education regarding slaughter and hygiene practices and dog population management and/or deworming initiatives using praziquantel $^{1}$. The development of vaccines targeting sheep intermediate hosts has now added a new tool to combat CE in highly impacted populations. The EG 95 vaccine has been tested in a number of countries, including China and Argentina with promising results ${ }^{45,46}$. Control program evaluation relies on surveillance for the pathogen in domestic dogs and ruminants, which is costly and laborintensive. Overall, the prevalence of human $\mathrm{CE}$ has been reduced in areas where long-term intensive control programs have been put in place. However, in most resource-poor areas, CE control remains a challenge?. Researchers are working to better understand the ecology of wildlife definitive (e.g., foxes and other wild canids) and intermediate (e.g., small mammals) hosts, to predict risks for human infection and improve anthelminthic baiting practices in rural and urban areas ${ }^{45}$.

\section{REFERENCES}

1. Budke CM, Casulli A, Kern P, Vuitton DA, Delabrousse E, Dentan C. Cystic and alveolar echinococcosis: Successes and continuing challenges. Hotez PJ, editor. PLoS Negl Trop Dis 2017-Apr

2. Brehm K, Koziol U. Echinococcus Host Interactions at Cellular and Molecular Levels. In 2017 [cited 2017 Jul 23]. p. 147212. Available from: http://linkinghub.elsevier.com/retrieve/pii/S0065308 X16300847

3. Lymbery AJ. Phylogenetic Pattern, Evolutionary Processes and Species Delimitation in the Genus
Echinococcus. Adv Parasitol. 2017; 95:11145.

4. Alvarez Rojas CA, Romig T, Lightowlers MW. Echinococcus granulosus sensu lato genotypes infecting humans review of current knowledge. Int $\mathrm{J}$ Parasitol. 2014 Jan

5. Gharbi HA, Hassine W, Brauner MW, Dupuch K. Ultrasound examination of the hydatic liver. Radiology. 1981;139(2):45963.

6. WHO Informal Working Group. International classification of ultrasound images in cystic echinococcosis for application in clinical and field epidemiological settings. Acta Trop. 2003 Feb

7. Brunetti E, Kern P, Vuitton DA. Expert consensus for the diagnosis and treatment of cystic and alveolar echinococcosis in humans. Acta Trop. 2010;114(1):116.

8. Bekhti A, Schaaps J, Capron M, et al. Treatment of hepatic hydatid disease with mebendazole: preliminary results in four cases. Br Med J 1977; 2:1047 1051 .

9. Saimot A, Meulemans A, Cremieux A, et al. Albendazole as a potential treatment for human hydatidosis. Lancet 1983; 2:652656.

10. Mueller P, Dawson S, Ferrucci JJ, Nardi G. Hepatic echinococcal cyst: successful percutaneous drainage. Radiology 1985; 155:627628.

11. Junghanss T, da Silva AM, Horton J, Chiodini PL, Brunetti E. Clinical management of cystic echinococcosis: state of the art, problems, and perspectives. Am J Trop Med Hyg. 2008;79(3):30111.

12. Khuroo MS, Wani N a, Javid G, Khan B a, Yattoo GN, Shah a H, et al. Percutaneous drainage compared with surgery for hepatic hydatid cysts. N Engl J Med. 1997;337(13):8817.

13. Gil-Grande L, Rodriguez-Caabeiro F, Prieto J et al. R controlled, Lancet trial of efficacy of albendazole in intra-abdominal hydatid disease., 342:12691272 1993.

14. Khuroo MS, Dar MY, Yattoo GN, Zargar SA, Javaid $\mathrm{G}$, Khan BA, et al. Percutaneous drainage versus albendazole therapy in hepatic hydatidosis: a prospective, randomized study. Gastroenterology 1993 May 
15. Keshmiri M, Baharvahdat H, Fattahi SH, Davachi B, Dabiri RH, Baradaran H, et al. Albendazole versus placebo in treatment of echinococcosis. Trans R Soc Trop Med Hyg. ;95(2):1904.

16. Shera TA, Choh NA, Gojwari TA, Shera FA, Shaheen FA, Wani GM, et al. A comparison of imaging guided double percutaneous aspiration injection and surgery in the treatment of cystic echinococcosis of liver. $\mathrm{Br} \mathrm{J}$ Radiol 2017 Apr

17. Kern P, Menezes da Silva A, Akhan O, Müllhaupt B, Vizcaychipi KA, Budke C, et al. The Echinococcoses. In 2017. p. 259369.

18. Menezes da Silva A. Hydatid cyst of the liver-criteria for the selection of appropriate treatment. Acta Trop. $2003 \mathrm{Feb}$

19. Taylor BR, Langer B. Current surgical management of hepatic cyst disease. Adv Surg. 1997

20. Daradkeh S, EL-Muhtaseb H, Farah G, Sroujieh AS, Abu-Khalaf M. Predictors of morbidity and mortality in the surgical management of hydatid cyst of the liver. Langenbeck's Arch Surg. 2007 Jan 18

21. Demirci S, Eraslan S, Anadol E, Bozatli L. Comparison of the results of different surgical techniques in the management of hydatid cysts of the liver. World J Surg. ;13(1):88-90-1.

22. Doğan R, Yüksel M, Cetin G, Süzer K, Alp M, Kaya S, et al. Surgical treatment of hydatid cysts of the lung: report on 1055 patients. Thorax. 1989 Mar 44(3):1929.

23. Liccardi G, Lobefalo G, Di Florio E, Di Iorio C, Occhiochiuso L, Romano L, et al. Strategies for the prevention of asthmatic, anaphylactic and anaphylactoid reactions during the administration of anesthetics and/or contrast media. J Investig Allergol Clin Immunol. 2008;18(1):111.

24. Isitmangil T, Sebit S, Tunc H, Gorur R, Erdik O, Kunter E, et al. Clinical experience of surgical therapy in 207 patients with thoracic hydatidosis over a 12year-period. Swiss Med Wkly. 2002 Oct 12 ;132(3738):54852.

25. Dziri C, Haouet K, Fingerhut A. Treatment of Hydatid Cyst of the Liver: Where Is the Evidence? World J Surg. 2004 Aug $3 ; 28(8): 7316$.

26. El Malki HO, El Mejdoubi Y, Souadka A, Mohsine R, Ifrine L, Abouqal R, et al. Predictive Factors of Deep
Abdominal Complications after Operation for Hydatid Cyst of the Liver: 15 Years of Experience with 672 Patients. J Am Coll Surg. 2008 Apr ;206(4):62937.

27. Gollackner B, Längle F, Auer H, Maier A, Mittlböck M, Agstner I, et al. Radical surgical therapy of abdominal cystic hydatid disease: factors of recurrence. World J Surg. 2000 Jun ;24(6):71721.

28. KAPAN M, KAPAN S, GOKSOY E, PEREK S, KOL E. Postoperative Recurrence in Hepatic Hydatid Disease. J Gastrointest Surg. 2006 May ;10(5):7349.

29. Lissandrin R, Agliata S, Brunetti E. Secondary peritoneal echinococcosis causing massive bilateral hydronephrosis and renal failure. Int J Infect Dis. 2013 Feb;17(2):e1412.

30. Dervisoglu A, Erzurumlu K, Taç K, Arslan A, Gürsel M, Hökelek M. Should intraoperative ultrasonography be used routinely in hepatic hydatidosis? Hepatogastroenterology. ; 49(47):13268.

31. World Health Organization (WHO) Informal Working Group of Echinococcosis. Puncture, Aspiration, Injection, Re-Aspiration. An option for the treatment of cystic echinococcosis, p. 140. Document WHO/CDS/CSR/SPH/2001.6. Geneva, Switzerland: WHO; 2001.pp. 1.

32. Men S, Yucesoy C, Edguer TR, Hekimoglu B. Percutaneous treatment of giant abdominal hydatid cysts: long-term results. Surg Endosc. 2006;20:16001606.

33. Ustunsoz B, Ugurel MS, Uzar AI, Duru NK. Percutaneous treatment of hepatic hydatid cyst in pregnancy: long-term results. Arch Gynecol Obstet. 2008 Jun ;277(6):54750.

34. Morris DL, Taylor DH. Optimal timing of postoperative albendazole prophylaxis in E. granulosus. Ann Trop Med Parasitol. 1988 Feb. ; 82(1):656.

35. Köroğlu M, Erol B, Gürses C, Türkbey B, Baş CY, Alparslan AŞ, et al. Hepatic cystic echinococcosis: Percutaneous treatment as an outpatient procedure. Asian Pac J Trop Med. 2014 Mar. ; 7(3):2125.

36. Neumayr A, Troia G, de Bernardis C, Tamarozzi F, Goblirsch S, Piccoli L, et al. Justified concern or exaggerated fear: the risk of anaphylaxis in percutaneous treatment of cystic echinococcosis-a systematic literature review. PLoS Negl Trop Dis. 
2011 Jun;5(6):e1154.

37. Horton J. Albendazole for the treatment of echinococcosis. Fundam Clin Pharmacol. 2003 Apr.;17(2):20512.

38. Rinaldi F, Brunetti E, Neumayr A, Maestri M, Goblirsch S, Tamarozzi F. Cystic echinococcosis of the liver: A primer for hepatologists. World J Hepatol. 2014 May 27 ; 6(5):293305.

39. Doğru D, Kiper N, Özçelik U, Yalçın E, Göçmen A. Medical treatment of pulmonary hydatid disease: for which child? Parasitol Int. 2005 Jun ;54(2):1358.

40. Vutova K, Mechkov G, Vachkov P, Petkov R, Georgiev P, Handjiev S, et al. Effect of mebendazole on human cystic echinococcosis: the role of dosage and treatment duration. Ann Trop Med Parasitol. 1999 Jun;93(4):35765.

41. Arif SH, Shams-ul-Bari, Wani NA, Zargar SA, Wani MA, Tabassum R, et al. Albendazole as an adjuvant to the standard surgical management of hydatid cyst liver. Int J Surg. 2008 Dec ; 6(6):44851.

42. Loos JA, Dávila VA, Rodrígues CR, Petrigh R, Zoppi JA, Crocenzi FA, et al. Metformin exhibits preventive and therapeutic efficacy against experimental cystic echinococcosis. CASULLI A, editor. PLoS Negl Trop Dis. 2017 Feb 9; 11(2):e0005370.

43. Bradley M, Horton J. Assessing the risk of benzimidazole therapy during pregnancy. Trans R Soc Trop Med Hyg. 2001 Jan ; 95(1):723.

44. Stojkovic M, Rosenberger KD, Steudle F, Junghanss T, Cretu C. Watch and Wait Management of Inactive Cystic Echinococcosis Does the Path to Inactivity Matter Analysis of a Prospective Patient Cohort. CASULLI A, editor. PLoS Negl Trop Dis. 2016 Dec $19 ; 10(12): \mathrm{e} 0005243$.

45. Craig PS, Hegglin D, Lightowlers MW, Torgerson PR, Wang Q. Echinococcosis. In 2017 . p. 55158. Available from: http://linkinghub.elsevier.com/ retrieve/pii/S0065308X16300859

46. Larrieu E, Mujica G, Gauci CG, Vizcaychipi K, Seleiman M, Herrero E, et al. Pilot Field Trial of the EG95 Vaccine Against Ovine Cystic Echinococcosis in Rio Negro, Argentina: Second Study of Impact. Garcia HH, editor. PLoS Negl Trop Dis. 2015 Oct 30 ; 9(10):e0004134. 